\title{
High-resolution macromolecular crystallography at the FemtoMAX beamline with time-over-threshold photon detection
}

Maja Jensen, ${ }^{a}{ }^{\ddagger}$ Viktor Ahlberg Gagnér, ${ }^{a}{ }^{\ddagger}$ Juan Cabello Sánchez, ${ }^{b}$ Åsa U. J.

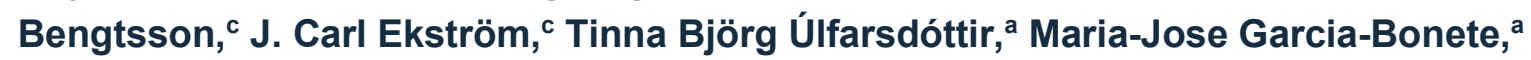
Andrius Jurgilaitis, ${ }^{d}$ David Kroon, ${ }^{d}$ Van-Thai Pham, ${ }^{d, e}$ Stefano Checcia, ${ }^{d}$ Hélène Coudert-Alteirac, ${ }^{\text {d }}$ Siawosch Schewa, ${ }^{f}$ Manfred Rössle, ${ }^{f}$ Helena Rodilla, ${ }^{b}$ Jan Stake, ${ }^{b}$ Vitali Zhaunerchyk, ${ }^{9}$ Jörgen Larsson ${ }^{\mathrm{c}}$ and Gergely Katona ${ }^{\mathrm{a}}{ }^{*}$

aDepartment of Chemistry and Molecular Biology, University of Gothenburg, Gothenburg, Sweden, 'bepartment of Microtechnology and Nanoscience, Chalmers University of Technology, Gothenburg, Sweden, 'Department of Physics, Lund University, PO Box 118, Lund, 22100, Sweden, 'MAX IV Laboratory, Lund University, PO Box 118, Lund, 22100, Sweden, e Center for Quantum Electronics, Institute of Physics, Vietnam Academy of Science and Technology, Hanoi, Vietnam, 'Technische Hochschule Lübeck, Lübeck, Germany, and 9Department of Physics, University of Gothenburg, Gothenburg, Sweden

* Correspondence email: gergely.katona@gu.se

¥ These authors contributed equally to this work.

Keywords: time-over-threshold; femtosecond; multilayer monochromator; macromolecular crystallography

Funding information: Rontgen-Angstrom Cluster Framework (award No. 2015-06099) 
Table S1 Extract from the XSCALE log file displaying the resolution dependent intensity statistics.

\section{$100 x$ data set}

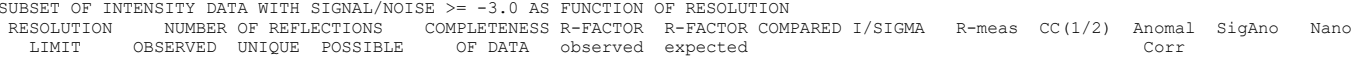

\begin{tabular}{|c|c|c|c|c|c|c|c|c|c|c|c|c|c|}
\hline 6.71 & 1333 & 421 & 461 & $91.3 \frac{8}{8}$ & $7.7 \%$ & $7.4 \frac{8}{8}$ & 1250 & 17.27 & $8.9 \%$ & $98.2 *$ & -9 & 0.818 & 151 \\
\hline 4.74 & 2832 & 765 & 784 & $97.6 \frac{2}{\frac{1}{0}}$ & $6.9 \%$ & $7.5 \frac{8}{8}$ & 2746 & 18.42 & $7.9 \%$ & $98.8^{*}$ & -4 & 0.777 & 357 \\
\hline 3.87 & 3260 & 944 & 984 & $95.9 \frac{8}{9}$ & $6.4 \%$ & $7.3 \%$ & 3139 & 18.29 & $7.4 \frac{8}{8}$ & $98.7 *$ & -15 & 0.672 & 402 \\
\hline 3.35 & 3629 & 1090 & 1152 & $94.6 \frac{2}{5}$ & $6.2 \%$ & $7.5 \%$ & 3489 & 16.88 & $7.1 \frac{8}{8}$ & $99.2 *$ & -18 & 0.643 & 424 \\
\hline 3.00 & 3772 & 1201 & 1289 & $93.2 \frac{2}{5}$ & $6.2 \%$ & $7.6 \%$ & 3629 & 15.03 & $7.3 \frac{8}{8}$ & $99.3^{*}$ & -13 & 0.700 & 388 \\
\hline 2.74 & 4182 & 1334 & 1430 & $93.3 \frac{2}{5}$ & $7.2 \frac{8}{6}$ & $8.1 \%$ & 4022 & 13.11 & $8.5 \frac{8}{0}$ & $99.0 *$ & -14 & 0.711 & 466 \\
\hline 2.54 & 4240 & 1397 & 1524 & $91.7 \frac{8}{8}$ & $7.8 \frac{8}{6}$ & $8.4 \frac{8}{8}$ & 4059 & 12.08 & $9.3 \frac{8}{8}$ & $98.8 *$ & -14 & 0.726 & 450 \\
\hline 2.37 & 4400 & 1496 & 1644 & $91.0 \frac{2}{8}$ & $8.5 \%$ & $9.1 \%$ & 4175 & 10.84 & $10.2 \frac{2}{0}$ & $98.4 *$ & -11 & 0.761 & 429 \\
\hline 2.24 & 4294 & 1521 & 1736 & $87.6 \frac{2}{8}$ & $8.9 \%$ & $9.4 \%$ & 4030 & 10.16 & $10.7 \%$ & $98.1^{*}$ & -10 & 0.765 & 381 \\
\hline 2.12 & 4422 & 1623 & 1828 & $88.8 \frac{2}{5}$ & $9.9 \%$ & $10.2 \frac{8}{\circ}$ & 4125 & 9.07 & $11.9 \%$ & $98.2^{\star}$ & -2 & 0.833 & 361 \\
\hline 2.02 & 4418 & 1702 & 1926 & $88.4 \frac{8}{5}$ & $10.9 \%$ & $11.6 \%$ & 4050 & 8.17 & $13.3 \frac{\circ}{\circ}$ & $97.8^{*}$ & -12 & 0.786 & 323 \\
\hline 1.94 & 4385 & 1765 & 2019 & $87.4 \frac{8}{5}$ & $12.8 \%$ & $13.3 \frac{5}{2}$ & 3960 & 7.16 & $15.6 \%$ & $97.0 *$ & -15 & 0.794 & 283 \\
\hline 1.86 & 4335 & 1825 & 2105 & $86.7 \frac{1}{2}$ & $15.2 \frac{8}{\circ}$ & $15.2 \frac{8}{\circ}$ & 3867 & 5.90 & $18.8 \%$ & $95.5 *$ & -11 & 0.773 & 240 \\
\hline $\begin{array}{l}1.79 \\
\text { S }\end{array}$ & 4228 & 1855 & 2173 & $85.4 \frac{8}{8}$ & $18.6 \%$ & 18.98 & 3704 & 4.92 & 23.38 & $94.1 *$ & -18 & 0.705 & 203 \\
\hline 1.73 & 4136 & 1897 & 2223 & $85.3 \frac{8}{9}$ & $22.1 \%$ & $22.8 \%$ & 3574 & 4.25 & 27.98 & $91.4 *$ & 5 & 0.825 & 163 \\
\hline $\begin{array}{l}1.68 \\
1.68\end{array}$ & 3953 & 1888 & 2321 & $81.3 \frac{8}{9}$ & $26.2 \%$ & $64.0 \%$ & $\begin{array}{r}3344 \\
3344\end{array}$ & $\begin{array}{l}3.49 \\
3.49\end{array}$ & $33.6 \%$ & $87.1^{\star}$ & 6 & 0.861 & $\begin{array}{l}134 \\
134\end{array}$ \\
\hline 1.63 & 3646 & 1827 & 2397 & $76.2 \frac{\circ}{\circ}$ & $31.0 \%$ & $36.8 \%$ & 2991 & 2.78 & $40.0 \div$ & $84.2^{\star}$ & -8 & 0.731 & 101 \\
\hline $\begin{array}{l}1.58 \\
\text { L }\end{array}$ & 3119 & 1682 & 2462 & $68.3 \frac{0}{5}$ & $35.6 \%$ & $38.2 \frac{5}{8}$ & 2421 & 2.31 & $46.5 \frac{1}{\square}$ & $77.3^{*}$ & 1 & 0.792 & 46 \\
\hline $\begin{array}{l}1.54 \\
\text { L }\end{array}$ & 2337 & 1418 & 2517 & $56.3 \frac{8}{8}$ & $45.8 \%$ & $58.7 \%$ & 1617 & 1.76 & $61.4 \frac{8}{8}$ & $61.6 *$ & -8 & 0.641 & 13 \\
\hline 1.50 & 1573 & 1079 & 2591 & $41.6 \frac{2}{5}$ & $47.5 \%$ & $80.6 \%$ & 906 & 1.44 & $64.5 \frac{8}{\sigma}$ & $60.3^{*}$ & -19 & 0.789 & 10 \\
\hline tal & 72494 & 28730 & 35566 & $80.8 \frac{8}{5}$ & $7.7 \%$ & $8.8 \%$ & 65098 & 8.00 & $9.1 \frac{8}{8}$ & $99.3 *$ & -10 & 0.747 & 5325 \\
\hline
\end{tabular}

$1 \mathrm{x}$ data set

SUBSET OF INTENSITY DATA WITH SIGNAL/NOISE $>=-3.0$ AS FUNCTION OF RESOLUTION

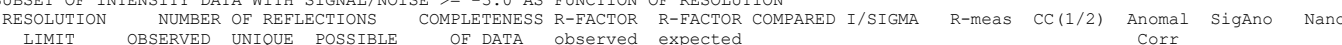

$\begin{array}{rrrr}9.39 & 597 & 169 & 181 \\ 6.64 & 1184 & 297 & 298 \\ 5.42 & 1568 & 359 & 363 \\ 4.70 & 1939 & 434 & 435 \\ 4.20 & 1950 & 470 & 479 \\ 3.83 & 2101 & 514 & 536 \\ 3.55 & 2222 & 550 & 569 \\ 3.32 & 2276 & 599 & 619 \\ 3.13 & 2335 & 625 & 647 \\ 2.97 & 2395 & 635 & 664 \\ 2.83 & 2567 & 694 & 718 \\ 2.71 & 2656 & 716 & 744 \\ 2.60 & 2673 & 740 & 773 \\ 2.51 & 2735 & 758 & 801 \\ 2.42 & 2726 & 781 & 825 \\ 2.35 & 2731 & 800 & 853 \\ 2.28 & 2738 & 817 & 887 \\ 2.21 & 2805 & 843 & 915 \\ 2.15 & 2724 & 853 & 930 \\ 2.10 & 2755 & 879 & 957 \\ \text { total } & 45677 & 12533 & 13194\end{array}$

\begin{tabular}{|c|c|c|c|c|}
\hline $93.4 \frac{8}{8}$ & $17.7 \frac{8}{8}$ & $24.5 \frac{8}{8}$ & 571 & 5.71 \\
\hline $99.7 \frac{}{8}$ & $18.2 \frac{\circ}{\circ}$ & $26.8 \frac{\circ}{\circ}$ & 1150 & \\
\hline $98.9 \%$ & $19.9 \%$ & $37.5 \%$ & 1545 & 5.42 \\
\hline $99.8 \%$ & $20.2 \frac{8}{5}$ & $32.7 \frac{8}{8}$ & 1920 & 5.58 \\
\hline $98.1 \frac{1}{6}$ & $18.9 \%$ & $29.8 \%$ & 1905 & 5.60 \\
\hline $95.9 \%$ & $20.1 \%$ & $36.0 \frac{8}{2}$ & 2053 & 5.27 \\
\hline $96.7 \frac{8}{8}$ & $22.5 \%$ & $44.7 \frac{8}{8}$ & 2167 & 4.76 \\
\hline $96.8 \%$ & $26.7 \%$ & $56.6 \%$ & 2211 & 4.14 \\
\hline $96.6 \%$ & $31.2 \%$ & $73.2 \%$ & 2264 & \\
\hline $95.6 \%$ & $37.0 \%$ & $92.7 \%$ & 2329 & 3.10 \\
\hline $96.7 \%$ & $44.2 \%$ & $122.8 \%$ & 2492 & 2.63 \\
\hline $96.2 \frac{2}{8}$ & $51.5 \frac{8}{8}$ & 126.7 움 & 2575 & 2.32 \\
\hline $95.7 \frac{8}{8}$ & $53.0 \%$ & $161.4 \frac{8}{\circ}$ & 2590 & 2.04 \\
\hline $94.6 \%$ & $59.1 \%$ & $183.7 \frac{\circ}{\circ}$ & 2648 & 1.80 \\
\hline $94.7 \frac{8}{8}$ & $67.7 \%$ & $205.0 \%$ & 2632 & 1.62 \\
\hline $93.8 \frac{8}{8}$ & $66.5 \%$ & $208.9 \%$ & 2618 & 1.51 \\
\hline $92.1 \frac{18}{8}$ & $70.3 \%$ & $209.8 \%$ & 2621 & 1.49 \\
\hline $92.1 \%$ & $75.2 \%$ & $213.1 \%$ & 2706 & 1.23 \\
\hline $91.7 \%$ & $77.2 \%$ & $248.8 \%$ & 2600 & 1.21 \\
\hline $91.8 \frac{8}{5}$ & $83.0 \%$ & 301.98 & 2635 & 1.06 \\
\hline
\end{tabular}

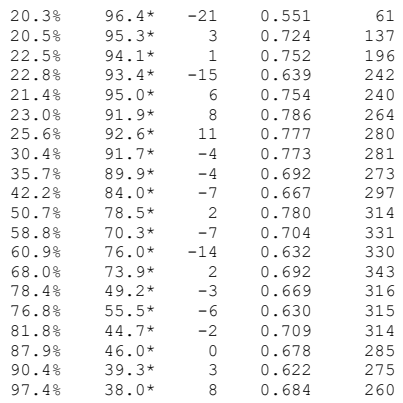

